\title{
Model-Based Feedback Control of High-speed Supercavitating Vehicles
}

\author{
Ziyao Cao \\ College of Marine Engineering \\ Northwestern Polytechnical University \\ Xi'an 710072, China \\ E-mail: caoziyao@nwpu.edu.cn
}

\begin{abstract}
Supercavitation is a means of drag reduction in water, wherein a body is enveloped in a gas layer in order to reduce skin friction. Depending on the type of supercavitating vehicle under consideration, the overall drag coefficient can be an order of magnitude less than that of a fully-wetted vehicle. However, even in its simplest conceptualization, supercavitating vehicle dynamics features slope-discontinuous force curves and time-delay effects, control presents special challenges. This paper has presented strategies for the control of the highly-coupled nonlinear system comprising a supercavitating vehicle. A three DOFs hydrodynamical model based on Newton's Laws was implemented for simulating the behavior of such a system. The linearization of the mathematical model is derived in detail. A Linear Quadratic Regulator (LQR) control design for the vehicle was formulated. Results of dynamical simulation for a specific vehicle were presented both for uncontrolled flight and with LQR-based feedforward-feedback control. The system behavior is dominated by the distinct change in the nature of the forces as the afterbody moves between a planing and non-planing condition, but that under the assumptions made for the three DOFs model the controller can eliminate the most undesirable behavior in either case.
\end{abstract}

Keywords: Supercavitation, Modeling, Linearization, LQR

\section{Introduction}

Supercavitation is a hydrodynamic process by which an undersea body is almost entirely enveloped in a layer of gas. Because the density and viscosity of the gas is dramatically lower than that of seawater, skin friction drag can be reduced by an order of magnitude. However, because the center of pressure is typically located well forward with respect to the center of gravity, control and maneuvering present special challenges not normally associated with fully-wetted vehicle dynamics 0 . These arise from the absence of certain physical effects such as lift on the body and from the presence of other effects such as the nonlinear interaction of the control surfaces and the body with the cavity boundary. Even if the body is stable inside the cavity, the vehicle may not be stable when in contact with the cavity. Nonlinear interaction of the control surfaces and the body with the cavity wall is very important when calculating the fin and planing forces acting on the vehicle. The cavity wall exerts a large restoring force over a short period of time. The nature of this instability forces the vehicle back into the cavity, often resulting in limit cycle behavior (Dzielski et al. 2003). Cavity-vehicle interaction also exhibits strong memory effects and cavity shape is a function of the history of the vehicle motion.

This paper investigates the control challenges associated with supercavitating vehicles using a low order, longitudinal axis vehicle model. In the first part of the paper, a detailed derivation of the equations of motion for the vehicle has been carried out using Newton's Laws. Various forces experienced by different regions of the vehicle have been explained. This model draws heavily on the benchmark HSSV model proposed by Dzielski and Kurdila (2003). That two DOFs model is extended to be three DOFs and include delay dependent vehicle-cavity interaction and a more refined planing model. The second part of the paper describes linearization of the equations of motion using small disturbance theory. It is observed that the linearization, even for a simple trim, straight-level flight, can be very complicated. Thus, numerical methods are used for this purpose. The third part of the paper formulates a Linear Quadratic Regulator (LQR) control design for the vehicle. A controller is synthesized to track pitch angle, angular rate, vertical position and vertical speed for the HSSV vehicle model using the proposed approach. Simulations of the closed-loop vehicle are performed and analyzed in the fourth section of the paper. Challenges facing the model creator and control designer are highlighted with respect to actuator and sensor requirements, modeling issues, robustness and performance. The paper concludes with a summary of the results and directions for future study. 


\section{Modeling of Supercavitating Vehicles}

Several mathematical descriptions of high speed supercavitating vehicles (HSSVs) are available in the literature (Dzielski et al. 2003) (Kirschner et al. 2003) (Kirschner et al. 2002). A one DOF longitudinal axis model was proposed to study the switching behavior of the supercavitating vehicle in (Kirschner et al. 2003). The model contains only longitudinal translational motion and its interaction with the cavity. The shape of the cavity boundary is described as a function of the time delayed position of the vehicle nose. A two DOFs longitudinal axis model for control design purposes was proposed in (Dzielski et al. 2003). The simplified model is linear within the cavity and nonlinear when the vehicle is in contact with the cavity (planing). The planing condition is defined as a function of vertical velocity and no time history dependency of the cavity is included. A high fidelity, nonlinear six DOFs mathematical model was proposed in (Kirschner et al. 2002). Lookup tables are used to calculate the forces generated by control surfaces as a function of immersion depth. The centerline of the cavity bubble is a function of the time history of the path of the vehicle nose affected by buoyancy.This paper extends the two DOFs longitudinal axis model developed in (Dzielski et al. 2003) to be three DOFs model and include the time dependent behavior of the cavity and redefines the planing condition to be a function of the vehicle vertical position, attack angle and time history of the cavity.

\subsection{Geometry Setup of the Vehicle}

The geometry of the vehicle being modeled is shown schematically in Figure 1. The geometry of the vehicle is intended to approximate the geometry of the actual vehicle. The body consists of a conical and a cylindrical section, where the cylindrical section is twice as long as the front conical section. The body length is denoted by $\boldsymbol{L}$ and its radius is $\boldsymbol{R}$. The body has uniform density $\rho_{b}$ (the body density is specified relative to the density of the surrounding fluid, $\rho_{b}=m \rho$, where $\rho$ is the density of sea water), from which the mass and inertia are calculated.

The reference point is at the center of gravity of the vehicle, hence the vehicle mass, $M$, the moment of inertial relative to the CG fixed reference frame, $I_{y y}$, can be expressed using the above definitions as:

$$
\begin{aligned}
& M=\frac{7}{9}(m \rho \pi) R^{2} L \\
& I_{y y}=\frac{11}{60} R^{4} L \rho \pi m+\frac{1891}{45360} R^{2} L^{3} \rho \pi m
\end{aligned}
$$

\subsection{Reference Frame Definition}

The reference frame used in modeling is shown clearly in Figure 1. The vehicle is assumed to be moving in an earth-fixed reference frame $O_{e} x_{e} z_{e}$, centered at any conveniently chosen point and described by the basis vector $\left(e_{x}, e_{z}\right)$. The $O_{e} z_{e}$ axis points in the downward direction, i.e., the direction of the gravity; the $O_{e} x_{e}$ axis is placed in the vertical plane and perpendicular to $O_{e} z_{e}$. A body-fixed frame $O x_{b} z_{b}$ is defined to describe rotation of body and described by the basis vector $\left(b_{x}, b_{z}\right)$. The frame $O x_{b} z_{b}$ is centered at $\mathrm{O}$, the center of gravity of the vehicle, and moves with the vehicle. The $O_{b} x_{b}$ axis is along the vehicle's symmetry line and points to the nose of vehicle; the $O_{b} z_{b}$ axis is perpendicular to $O_{b} x_{b}$ and points downward.

The transformation matrix from $\boldsymbol{B}$ to $\boldsymbol{E}$ can be written as in

$$
\left[\begin{array}{l}
e_{x} \\
e_{z}
\end{array}\right]=\left[\begin{array}{cc}
\cos \theta & \sin \theta \\
-\sin \theta & \cos \theta
\end{array}\right]\left[\begin{array}{l}
b_{x} \\
b_{z}
\end{array}\right]
$$

Where, $\theta$ is pitch angle of the vehicle.

\subsection{Kinematical Equations}

To apply Newton's Laws, acceleration of the CG is required. The translational acceleration can be calculated by time differentiation of $\boldsymbol{V}$ in Newtonian (inertial) frame. A differentiation formula can be used to find the time derivative, in some frame, for a vector defined in some other related frame.

$$
\left.\frac{d}{d t}(\mathbf{v})\right|_{I}=\left.\frac{d}{d t}(\mathbf{v})\right|_{B}+{ }^{I} \omega^{B} \times \mathbf{v}
$$

Where, subscript $\boldsymbol{I}$ denotes Newtonian (inertial) frame, and $\mathbf{B}$ is the body-fixed frame. ${ }^{I} \omega^{B}$ is angular velocity of the body (or body-fixed frame) in the $\boldsymbol{I}$ frame, $\boldsymbol{v}$ is the velocity in $\boldsymbol{I}$ frame, of the point where acceleration is desired. Using the formula the acceleration of $\mathbf{C G}$ of vehicle in $\mathbf{E}$ frame can be obtained ( $w$ vertical speed, $u$ horizontal speed, $q$ pitch rate):

$$
\left\{\begin{array}{l}
A x_{e}=\dot{u}+q w \\
A z_{e}=\dot{w}-q u
\end{array}\right.
$$


Similarly, the rotational acceleration will be required in the frame $\boldsymbol{E}$. For the model is 3DOFs in vertical plane, this rotational acceleration is simply $\dot{q}$.

Now that the accelerations of various parts of the torpedo are known, Newton's Laws can be used to derive the dynamic equations of motion. Newton's Laws state that the rate of change of momentum is equal to the sum of external force applied on the body, which can also be extended to rotation. Then, the dynamic equations of motion can be obtained from Newton's Laws by an assumption that the mass of the vehicle is constant, this assumption is valid for a short period of time. The equations are:

$$
\left\{\begin{array}{l}
m(\dot{u}+q w)=\sum F_{x} \\
m(\dot{w}-q u)=\sum F_{z} \\
I_{y y} \dot{q}=\sum M
\end{array}\right.
$$

\subsection{Forces on the Vehicle}

It is assumed that when the vehicle body is fully contained in the cavity, the only hydrodynamic forces acting are due to the cavitator, immersed fins and planing.

2.4.1 Forces on Cavitator and Fins

Assuming that the cavitator drag coefficient is modeled as $C_{x}=C_{x 0}(1+\sigma)$, where $\sigma$ is the capitation number and $C_{x 0}=0.82$ (Logvinovich, 1972), the resulting force on the cavitator is approximately:

$$
F_{c a v}=\frac{1}{2} \pi \rho R_{n}^{2} C_{x} V^{2} \alpha_{c}=C_{l} V^{2} \alpha_{c}
$$

In the above equation, $C_{l}=\pi \rho R_{n}^{2} C_{x} / 2, R_{n}$ is the cavitator radius, $\rho$ sea water density, $V$ vehicle velocity, $\alpha_{c}$ denotes the angle-of-attack due to cavitator deflection $\delta_{c}$ and vertical body velocity.

The total force acting on an equivalent control surface fixed at the tail of the vehicle is modeled in relation to the cavitator force:

$$
F_{\text {fin }}=-n C_{l} V^{2} \alpha_{f}
$$

Where, the parameter $n$ represents the effectiveness of the control surfaces in provided lift as a function of angle-of-attack relative to the cavitator effectiveness.

In both force equations, the angle-of-attack terms are assumed to be small so that small angle approximations apply to trigonometric functions. Assuming the fins are located at the transom of the vehicle and have a moment arm $L_{f}$ and the cavitator has a moment arm $L_{c}$ relative to the reference point for the equation of motion, the total angle-of-attack equations are:

$$
\begin{aligned}
& \alpha_{c}=\frac{w-q L_{c}}{V}+\delta_{c} \\
& \alpha_{f}=\frac{w+q L_{f}}{V}+\delta_{f}
\end{aligned}
$$

Where, $\quad L_{c}=\frac{17}{28} L$ and $L_{f}=\frac{11}{28} L$

Then, the forces and moments due to cavitator and fins can be calculated as:

$$
\begin{aligned}
& \left\{\begin{array}{l}
F_{x}^{c a v}=F_{c a v} \cos \delta_{c} \\
F_{z}^{c a v}=F_{c a v} \sin \delta_{c} \\
M_{c a v}=F_{x}^{c a v} L_{c}
\end{array}\right. \\
& \left\{\begin{array}{l}
F_{x}^{f i n}=F_{f i n} \cos \delta_{f} \\
F_{z}^{f i n}=F_{f i n} \sin \delta_{c} \\
M_{f i n}=F_{x}^{f i n} L_{f}
\end{array}\right.
\end{aligned}
$$




\subsubsection{Planing Force and Moment}

In the original two DOFs model proposed in (Dzielski et al. 2003), the interaction of the vehicle and cavity was not a function of the cavitator position. Hence it was not delay dependent. In the extended model, the planing condition is a function of the cavity boundary and a delayed function of the cavitator position. The force and moment generated by planing are approximated by Paryshev's method (Paryshev, 2002).

$$
\begin{aligned}
& \quad F_{p}\left(h^{\prime}, \alpha_{i m}\right)=-C_{p}\left(1-\left(\frac{R^{\prime}}{h^{\prime}(t)+R^{\prime}}\right)^{2}\right)\left(\frac{1+h^{\prime}(t)}{1+2 h^{\prime}(t)}\right) \alpha_{i m}(t) \\
& M_{p}=F_{p} \times L_{f}
\end{aligned}
$$

The unknown variables are the following: $C_{p}$ is a coefficient has value $\pi \rho R^{2} V^{2} ; h^{\prime}(t)$ is the normalized immersion depth, the amount with which the body penetrates the water; $\alpha_{i m}(t)$ the immersion angle, angle between the body's surface and the cavity; $R^{\prime}=\left(R_{c}-R\right) / R$ normalized median distance between the transom and the cavity. The $h^{\prime}(t)$ and $\alpha_{i m}(t)$ are functions of time, because they are delay dependent, the time delay between the fluid impinging on the cavity and the aft end of the vehicle is $\tau=L / V$. The time dependence of the states and controls is suppressed in the paper unless it is needed for clarity.

The planing depth is a discontinuous functions, can be represented as:

$$
h^{\prime}=\left\{\begin{array}{l}
{\left[z(t)+\theta L+R-z(t-\tau)-R_{c}\right] / R} \\
\text { if } \quad z(t-\tau)+R_{c}<z(t)+\theta L+R \\
0 \quad \text { inside cavity } \\
{\left[z(t)-\theta L+R-z(t-\tau)-R_{c}\right] / R} \\
\text { if } \quad z(t-\tau)-R_{c}<z(t)+\theta L+R
\end{array}\right.
$$

The position of the vehicle transom is described by the position of the vehicle nose plus a term caused by the vehicle angle radius at the transom. The cavity's boundary is located at $R_{c}$ distance from the cavity centerline position $z(t-\tau)$, the immersion is the difference between the two. The immersion angle is also determined by the planing location:

$$
\alpha_{i m}= \begin{cases}\theta-\frac{w(t-\tau)}{V}-\frac{\dot{R}_{c}}{V} & \text { (bottom contact) } \\ 0 & \text { (no contact) } \\ \theta-\frac{w(t-\tau)}{V}+\frac{\dot{R}_{c}}{V} & \text { (top contact) }\end{cases}
$$

Where $\dot{R}_{c}$ denotes the contribution from the fact that the cavity radius is usually contracting in the region of the planing location and this can have a significant effect on the apparent angle-of-attack.

To simplify the following equations and assuming that the body planes at the approximate location of the fins $L$, two numerical constants are defined by:

$$
\begin{aligned}
& k_{1}=\frac{L}{R_{n}}\left(\frac{1.92}{\sigma}-3\right)^{-1}-1 \\
& k_{2}=\left(1-\left(1-\frac{4.5 \sigma}{1+\sigma}\right) k_{1}^{(40 / 17)}\right)^{1 / 2}
\end{aligned}
$$

The expression for the radius of the cavity at a distance $\mathrm{L}$ from the cavitator is

$$
R_{c}=R_{n}\left(0.82 \frac{(1+\sigma)}{\sigma}\right)^{1 / 2} k_{2}
$$

And the expression for the contraction rate of the cavity $\dot{R}_{c}$ is

$$
\dot{R}_{c}=-\frac{20}{17}\left(0.82 \frac{1+\sigma}{\sigma}\right)^{1 / 2} V\left(1-\frac{4.5 \sigma}{1+\sigma}\right) k_{1}^{(23 / 17)} /\left(k_{2}\left(\frac{1.92}{\sigma}-3\right)\right)
$$

The equations for the cavity shape that are presented here are valid only if the following constraint on the length is 
satisfied:

$$
L>R_{n}\left(\frac{1.92}{\sigma}-3\right)
$$

The above equations describe the dynamic behavior of an HSSV; these equations must be complemented with a $\mathrm{s}$ kinematics equation for depth in frame $\boldsymbol{E}$

$$
\dot{z}=-u \sin \theta+w \cos \theta
$$

Then, substituting equations about forces and moments (11) (14) into equation(6), the equation of motion for the supercavitating vehicles restricted to the vertical plane become:

$$
\left\{\begin{array}{l}
\dot{z}=-u \sin \theta+w \cos \theta \\
\dot{u}=\left(F_{x}^{c a v}+F_{x}^{f i n}\right) / M-q w-g \sin \theta \\
\dot{w}=\left(F_{z}^{c a v}+F_{z}^{f i n}\right) / M+q u+g \cos \theta \\
\dot{q}=\left(F_{z}^{c a v} L_{c}+F_{z}^{f i n} L_{f}+F_{p} L_{f}\right) / I_{y y} \\
\dot{\theta}=q
\end{array}\right.
$$

\section{Control Design of Hssvs}

\subsection{Linearization}

The equations of motion, as in the case of a supercavitating vehicle, are represented by a set of first-order differential equations.

$$
\dot{\mathbf{x}}=\mathbf{f}(\mathbf{x}, \mathbf{u})
$$

Using $\mathbf{f}: \square^{n} \rightarrow \square^{n}$ as a nonlinear function of a time-varying vector $\mathbf{x} \in \square^{n}$ and $\mathbf{u} \in \square^{n}$. For control design, the system dynamics are observed at some trim conditions by giving perturbations to states of the system at that trim. The dynamics associated with these perturbations are obtained by linearization. An advantage by linearization is that most of the control methodology is based on linear equations of motion. A controller is designed initially for the linear system and then tested for the actual nonlinear system.

We determine the linearization for the planar vehicle about a steady state. We chose five states, let $\mathbf{x}=(z, u, w, q, \theta)^{T}$ and let $\mathbf{u}=\left(\delta_{c}, \delta_{f}\right)^{T}$. Assuming the operating point is $\mathbf{x}_{0}=\left(z_{0}, u_{0}, w_{0}, q_{0}, \theta_{0}\right)^{T}$ and $\mathbf{u}_{0}=\left(\delta_{c 0}, \delta_{f 0}\right)^{T}$, Define

$$
\begin{aligned}
\Delta \mathbf{x} & =\mathbf{x}-\mathbf{x}_{0} \\
\Delta \mathbf{u} & =\mathbf{u}-\mathbf{u}_{0}
\end{aligned}
$$

Then the linearized system is

$$
\Delta \dot{\mathbf{x}}=A \Delta \mathbf{x}+B \Delta \mathbf{u}
$$

Where A and B are defined as shown in (26) and (27). Here:

$$
\begin{gathered}
\alpha_{u}^{c}=\alpha_{u}^{f}=-\frac{w_{0}}{V_{0}^{2}} \quad \alpha_{w}^{c}=\alpha_{w}^{f}=\frac{u_{0}}{V_{0}^{2}} \\
\alpha_{q}^{c}=\frac{-L_{c}}{V_{0}} \quad \alpha_{q}^{f}=\frac{L_{f}}{V_{0}} \\
F_{u}^{c a v}=C_{l}\left(2 u_{0} \alpha_{u}^{c}-w_{0}\right) \\
F_{w}^{c a v}=C_{l}\left(2 w_{0} \alpha_{w}^{c}+u_{0}\right) \\
F_{u}^{f i n}=-n C_{l}\left(2 u_{0} \alpha_{u}^{f}-w_{0}\right) \\
F_{w}^{f i n}=-n C_{l}\left(2 w_{0} \alpha_{w}^{f}+u_{0}\right) \\
F_{q}^{c a v}=-\frac{C_{l} L_{c}}{V_{0}} \quad F_{q}^{f i n}=-\frac{n C_{l} L_{f}}{V_{0}}
\end{gathered}
$$


Where we have abbreviated $\partial \alpha_{c} / \partial u$ as $\alpha_{u}^{c}$, etc., and

$$
\begin{aligned}
& a_{15}=-u_{0} \cos \theta_{0}-w_{0} \sin \theta_{0} \\
& a_{22}=\left(F_{u}^{c a v} \cos \delta_{c 0}+F_{u}^{f i n} \cos \delta_{f 0}\right) / M \\
& a_{23}=\left(F_{w}^{c a v} \cos \delta_{c 0}+F_{w}^{f i n} \cos \delta_{f 0}\right) / M-q_{0} \\
& a_{24}=\left(F_{q}^{c a v} \cos \delta_{c 0}+F_{q}^{\text {fin }} \cos \delta_{f 0}\right) / M-w_{0} \\
& a_{32}=\left(F_{u}^{c a v} \sin \delta_{c 0}+F_{u}^{f i n} \sin \delta_{f 0}\right) / M+q_{0} \\
& a_{33}=\left(F_{w}^{c a v} \sin \delta_{c 0}+F_{w}^{f i n} \sin \delta_{f 0}\right) / M \\
& a_{34}=\left(F_{q}^{c a v} \sin \delta_{c 0}+F_{q}^{f i n} \sin \delta_{f 0}\right) / M+u_{0} \\
& a_{42}=\left(F_{u}^{c a v} L_{c} \cos \delta_{c 0}+F_{u}^{f i n} L_{f} \cos \delta_{f 0}\right) / I_{y y} \\
& a_{43}=\left(F_{w}^{c a v} L_{c} \cos \delta_{c 0}+F_{w}^{f i n} L_{f} \cos \delta_{f 0}\right) / I_{y y} \\
& a_{44}=\left(F_{q}^{c a v} L_{c} \cos \delta_{c 0}+F_{q}^{f i n} L_{f} \cos \delta_{f 0}\right) / I_{y y} \\
& A=\left[\begin{array}{ccccc}
0 & -\sin \theta_{0} & \cos \theta_{0} & 0 & a_{15} \\
0 & a_{22} & a_{23} & a_{24} & -g \cos \theta_{0} \\
0 & a_{32} & a_{33} & a_{34} & -g \sin \theta_{0} \\
0 & a_{42} & a_{43} & a_{44} & 0 \\
0 & 0 & 0 & 1 & 0
\end{array}\right] \\
& B=\left[\begin{array}{cc}
0 & 0 \\
-\left.F_{c a v}\right|_{x_{0}} \sin \delta_{c 0} & -\left.F_{f i n}\right|_{x_{0}} \sin \delta_{f 0} \\
\left.F_{c a v}\right|_{x_{0}} \cos \delta_{c 0} & \left.F_{f i n}\right|_{x_{0}} \sin \delta_{f 0} \\
\frac{-F_{c a v} \mid x_{x_{0}} \sin \delta_{c 0}}{I_{y y}} & \frac{-\left.F_{f i n}\right|_{x_{0}} \sin \delta_{f 0}}{I_{y y}} \\
0 & 0
\end{array}\right]
\end{aligned}
$$

The notation $\left.(\bullet)\right|_{x_{0}}$ indicates that the quantity is to be evaluated at $x_{0}$. This linearization can be used to check features of a given vehicle design, e.g., to check stability or controllability of a desired motion mode given a choice of vehicle design parameters. One can also use this linearization to help automate the design procedure.

\subsection{Control Design Setup}

There are various control methods, like linear quadratic regulator (LQR) synthesis, $\mu$-synthesis etc., which can be used to design a controller. Each of these methods has advantages and disadvantages.

LQR method gives a constant gain controller which considers the problem of robustness only in terms of gain and phase margins. It is a standard linear optimal control design method which produces a stabilizing control law that minimizes a cost function that is a weighted sum of the squares of the states and input variables. We assume that all of the states are available for feed back. If this were not the case, then, it is possible to design an estimator to determine the unmeasured states.

It is assumed that knowledge of the system state is accurate, ignoring high-frequency hydrodynamic noise excitation and other noise sources. However, the nonlinearity of the vehicle-cavity system has been retained. Nonlinearity enters the system via the vehicle planing behavior, due to the nonlinear force coefficients and to memory effects associated with cavity evolution. Control has been implemented via LQR. Cavity memory effects and the discontinuities in the force coefficients require incorporation of the feedforward model to maintain acceptable system performance. The current investigation focused on the simplified system is shown in Figure 2.

For the simplified system, the input to the LQR controller is the error vector, $\Delta x=x_{d}-x_{a}$, computed as the difference between the commanded state vector, $x_{d}$, and the corresponding actual state vector, $x_{a}$. Both the actual and commanded state vectors have the form: $\{z, u, w, q, \theta\}^{T}$. A classical regulator would provide a control vector, $\boldsymbol{u}$, directly 
to the hydrodynamic control effectors (here, the fins and cavitator): $\mathbf{u}=\left(\delta_{c}, \delta_{f}\right)^{T}$. However, the control vector of interest involves both the feedback output form the LQR, $\mathbf{u}_{r}$, and output from the feedforward model, $\mathbf{u}_{f}: \mathbf{u}=\mathbf{u}_{r}+\mathbf{u}_{f}$. The feedforward model investigated in our work is specialized for supercavitating vehicle planing dynamics. A scheme has been selected about one of two distinct fixed points in straight flight:

$$
u_{f} \in\left\{\begin{array}{l}
u_{1} \text { fin supported, no planing } \\
u_{2} \text { planing, zero fin deflection }
\end{array}\right.
$$

The point selected depends on the planing operating condition.

Regulation for the general LQR is measured by the quadratic performance criterion

$$
J(\mathbf{u})=\int_{t=0}^{\infty}\left(\Delta \mathbf{x}^{T} \mathbf{Q} \Delta \mathbf{x}+2 \Delta \mathbf{x}^{T} \mathbf{N} \mathbf{u}_{r}+\mathbf{u}_{r}{ }^{T} \mathbf{R} \mathbf{u}_{r}\right) d t
$$

Where the user-specified weighting matrices, $\boldsymbol{Q}, \boldsymbol{N}$, and $\boldsymbol{R}$, define the trade-off between regulation performance and control effort. Once the state and control matrices have been obtained, the main variables that the LQR controller depends on are the weighting matrices $\boldsymbol{Q}, \boldsymbol{R}$ and $\boldsymbol{N}$. In this case the cross coupling matrix $\boldsymbol{N}$ is chosen to be zero. The matrices $\boldsymbol{Q}$ and $\boldsymbol{R}$ penalize the cost function for higher state and control values respectively. A higher value in $\boldsymbol{Q}$ matrix would cause a better tracking. A larger $\boldsymbol{R}$ would constrain the control surface deflection. An optimum combination of the matrices is obtained iteratively, so as to get good tracking with achievable control deflections.

A MATLAB ${ }^{\mathrm{B}}$ control system Toolbox routine is used to determine the gain matrices, $\boldsymbol{K}_{\boldsymbol{s}}$, which minimize the cost function. $\boldsymbol{J}(\boldsymbol{u})$, for use in the state feedforward-feedback control law (MATH WORKS, 1998) for each of the two fixed points considered. The control law is then expressed as:

$$
\mathbf{u}=\mathbf{u}_{f s}-K_{s} \mathbf{x} \quad s \in\{1,2\}
$$

A substantial portion of the current work is devoted to testing various combinations of the weighting matrices $\boldsymbol{Q}$ and $\boldsymbol{R}$ to achieve acceptable performance. The weighting and gain matrices are all computed prior to flight simulation.

\section{Results of Simulations}

The specific set of parameter values used in our simulations is as follows (Dzielski et al. 2003 ): $g=9.81 \mathrm{~m} / \mathrm{s} 2, m=2, R_{n}=$ $0.0191 \mathrm{~m}, R=0.0508 \mathrm{~m}, L=1.8 \mathrm{~m}, V_{0}=75 \mathrm{~m} / \mathrm{s}, \sigma=0.03, n=0.5, C_{x 0}=0.82$.

We control the vehicle to maintain a desired operating point:

$(\bar{z}, \bar{u}, \bar{w}, \bar{q}, \bar{\theta})=(0,75,0,0,0)$

The matrices for state tracking are given as follow:

$\mathbf{Q}=\operatorname{diag}([10,0,1,0,0.5])$

$\mathbf{R}=\operatorname{diag}([3,1])$

The simulation results are shown in Figure 3 and Figure 4. We can see that, in short period of time (one second), the uncontrolled case started with violent tail-slap behavior and undergone a limit cycle oscillations. This could be explained as that the transom of vehicle without control was fast immersed in the bottom of the cavity, the resulting planing force acts in the opposite direction of the immersion, providing a large force to bounce the body back into the cavity, leading to oscillatory motion like a fast boat bouncing on the top of water.

More interestingly, the results in (Dzielski et al. 2003) indicated that the two DOFs model system had no equilibria in erither region, only the closed-loop system with control had equilibrium point., whereas the motion of our three DOFs system in uncontrolled case is a kind of attenuation oscillation around a equilibrium point:

$$
(\bar{z}, \bar{u}, \bar{w}, \bar{q}, \bar{\theta})=(0.09229,74.848,0.1229,0,0.0251)
$$

We can change our points of view into kinetic energy: the horizontal speed of two DOFs model was restricted to constant, the energy contributed by planing force accumulated continuously and resulted in non-limit motion. For our three DOFs model, since there is no propulsion in the system, the horizontal speed is reduced extremely, so the sum kinetic energy of the vehicle system is decreasing and a limit cycle oscillation motion can be obtained..

As shown in Figure 4, the uncontrolled case is dominated by a continuous skipping mode, whereas our controller eliminates this mode. The planing force history indicates that skipping occurs in both cases - apparently the controlled response during the planing portion of the vehicle is sufficient to mitigate the unsteady cavity behavior in such a manner that the afterbody excursions are not so extreme. 


\section{Summary}

This paper describes the control challenges associated with supercavitating vehicles. The feedback control problem features a delay dependent system with significant nonlinearities affecting the design. There are a number of open questions to be resovled before such a vehicle can be successfully controlled. These include:

- The current model assumes all the states are available for the feedback. A detailed study of the sensors is required. The available sensors and their characteristics affect the actual performance of the controller.

- The LQR controllers obtained are typically known as the 'inner-loop' controllers. An outer-loop controller is also needed for guidance and navigation.

- Robustness of feedback controller to modeling errors and poor physical models.

The performance and robustness requirements facing the design of a feedback controller will significantly shape the design of future supercavitating vehicles.

\section{References}

ANUKUL GOEL. (2005). Robust control of supercavitating vehicles in the presence of dynamic and uncertain cavity. Florida:University of Florida.

Arndt REA, Balas GJ, Wosnik M. (2005). Control of cavitating flows: A perspective. Japan Society of Mechanical Engineers International Journal, Japan.

B.Vanek, J.Bokor, and G.J.Balas. (2006). Developmets in high-speed underwater vehicle control. J.Vibration and Control.

B.Vanek, J.Bokor, and G.J.Balas. (2006). Theoretical aspects of high-speed supercavitation vehicle control. American Control Conference. Minneapolis.

Ivan N.Kirschner, James S.Uhlman. (2006). Overview of High-speed supercavitating vehicle control. AIAA Guidance, Navigation and Control Conference and Exhibit. Keystone, Colorado.

John Dzielski, Andrew Kurdila. (2003). A benchmark control problem for supercavitating vehicles and an initial investigation of solutions . Journal of Vibration and control.vol.9,no,pp.791-804.

Kirschner IN, Kring DC, Stokes AW, Uhlman JS. (2002). Control strategies for supercavitating vehicles. Vibration and Control 8:219-242.

Kirschner IN, Rosenthal B.J, Uhlman JS. (2003). Simplified dynamical system analysis of supercavitating high-speed bodies. Fifth International Symposium on Cavitation, Osaka Japan.

Logvinovigh GV. (1972). Hydrodynamics of free-boundary flows. NASA-TT-F-658, US Departmet of Commerce, Washington D.C.

Paryshev EV. (2002). The plane problem of immersion of an expanding cylinder through a cylindrical free surface of variable radius. International Summer Scientific School on High-Speed Hydrodynamics, Cheboksary, Russia.

Savchenko Yu N. (2001). Control of Supercavitating Motion of Bodies, VKI Special Course on Supercavitating Flows, Brussels: RTO-AVT and VKI. RTO-EN-010(11).

Shao Y, Mesbahi M, Balas GJ. (2003). Planing, switching and supercavitating flight control. AIAA Guidance, Navigation and Control Conference, AIAA-2003-5724. 


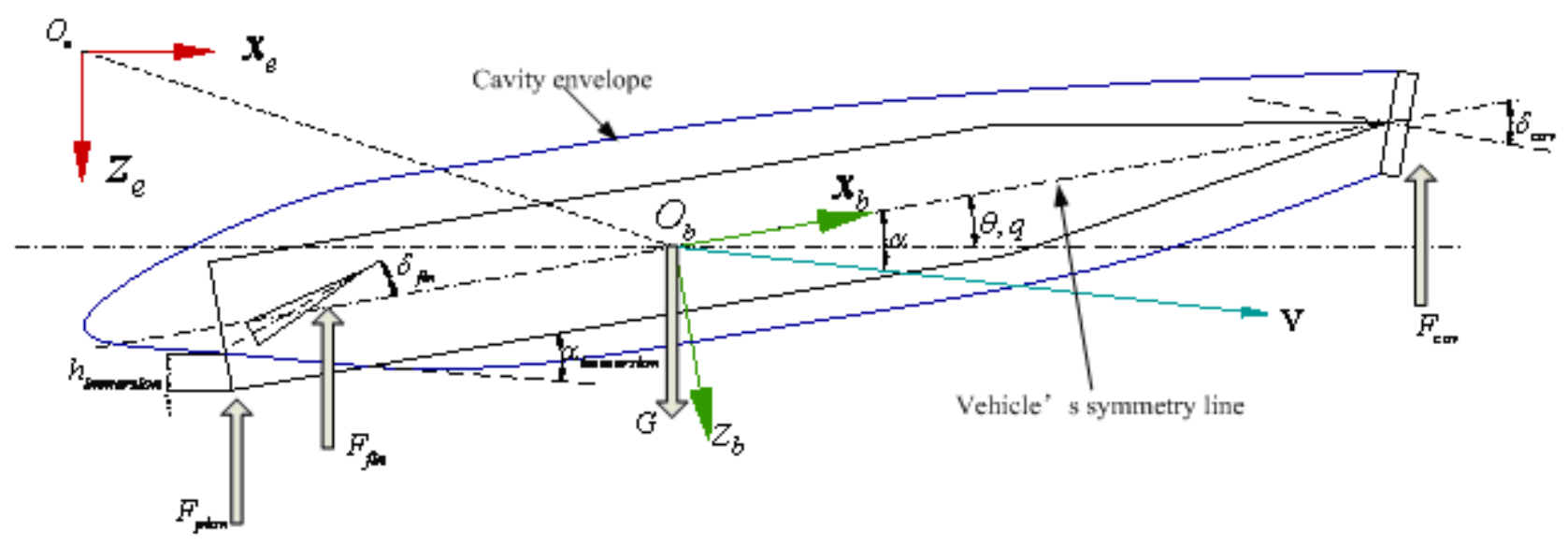

Figure 1. Schematic diagram of 3 DOFs model

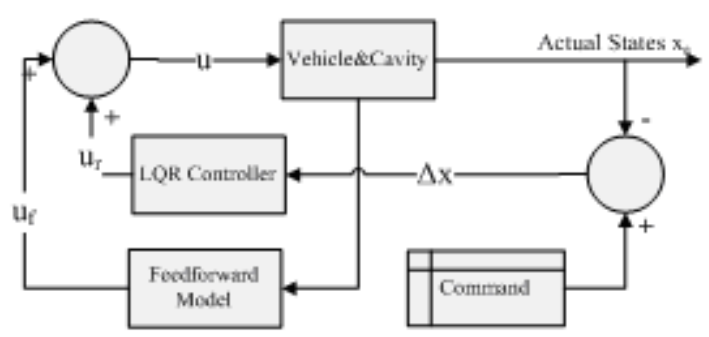

Figure 2. Simplified dynamical systems model of a supercavitating vehicle
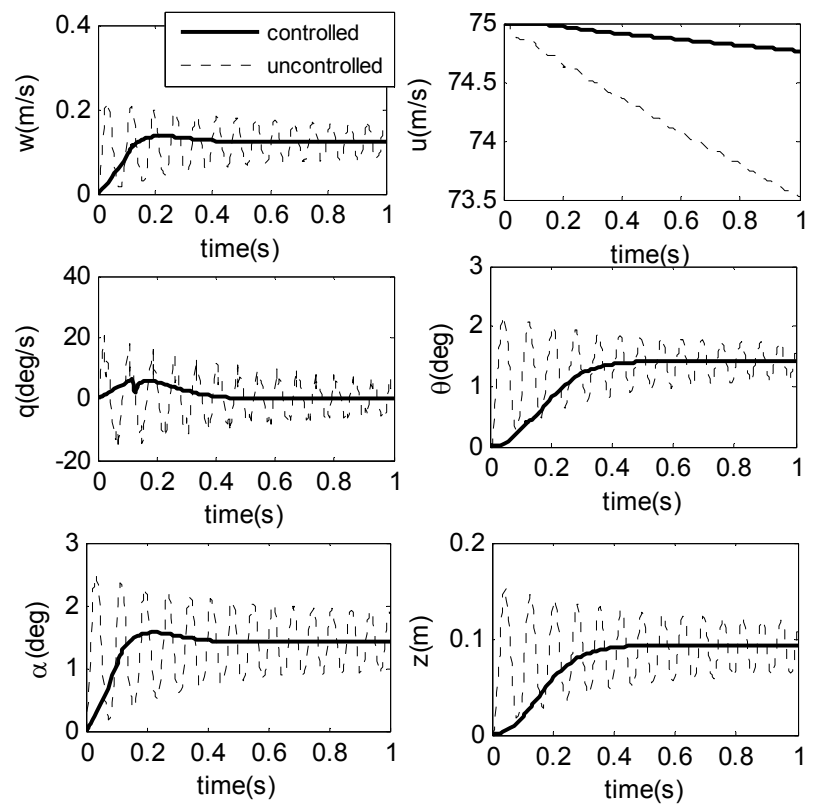

Figure 3. Simulation results of HSSV (w,u,q, $,, \alpha, z)$ 

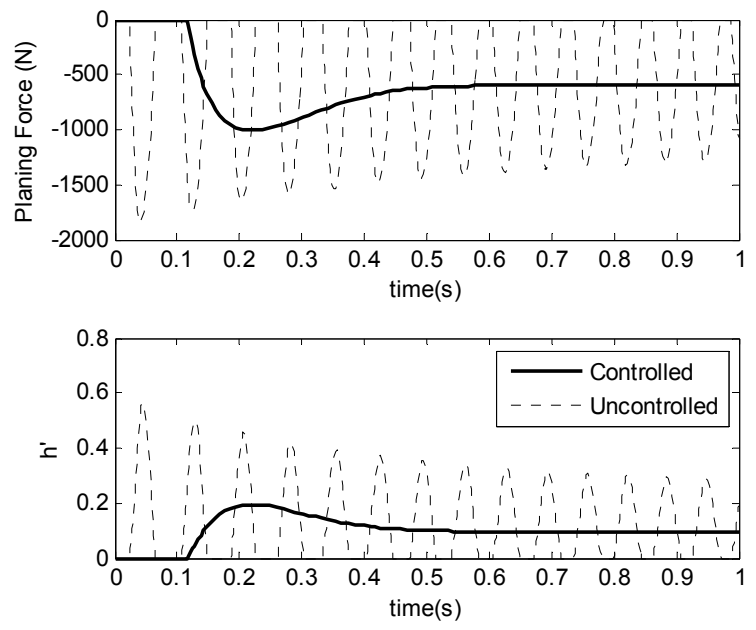

Figure 4. Simulation results of HSSV (Fp, h') 